\title{
Optimal Virtual Monochromatic Images for Evaluation of Normal Tissues and Head and Neck Cancer Using Dual-Energy CT
}

\author{
S. Lam, R. Gupta, M. Levental, E. Yu, H.D. Curtin, and R. Forghani
}

\begin{abstract}
BACKGROUND AND PURPOSE: Dual-energy CT is not used routinely for evaluation of the head and neck, and there is no consensus on the optimal virtual monochromatic image energies for evaluating normal tissues or head and neck cancer. We performed a quantitative evaluation to determine the optimal virtual monochromatic images for visualization of normal tissues, head and neck squamous cell carcinoma, and lymphadenopathy.
\end{abstract}

MATERIALS AND METHODS: Dual-energy CT scans from 10 healthy patients and 30 patients with squamous cell carcinoma were evaluated at different virtual monochromatic energy levels ranging from 40 to $140 \mathrm{keV}$. The signal-to-noise ratios of muscles at 6 different levels, glands (parotid, sublingual, submandibular, and thyroid), 30 tumors, and 17 metastatic lymph nodes were determined as measures of optimal image quality. Lesion attenuation and contrast-to-noise ratios (compared with those of muscle) were evaluated to assess lesion conspicuity.

RESULTS: The optimal signal-to-noise ratio for all the tissues was at $65 \mathrm{keV}(P<.0001)$. However, tumor attenuation $(P<.0001)$, attenuation difference between tumor and muscles $(P=.03)$, and lesion contrast-to-noise ratios $(P<.0001)$ were highest at $40 \mathrm{keV}$.

CONCLUSIONS: The optimal image signal-to-noise ratio is at $65 \mathrm{keV}$, but tumor conspicuity compared with that of muscle is greatest at $40 \mathrm{keV}$. Optimal evaluation of the neck may be best achieved by a multiparametric approach, with $65-\mathrm{keV}$ virtual monochromatic images providing the best overall image quality and targeted use of $40-\mathrm{keV}$ virtual monochromatic images for tumor evaluation.

ABBREVIATIONS: CNR = contrast-to-noise ratio; DECT = dual-energy CT; HNSCC = head and neck squamous cell carcinoma; keV = kiloelectron volt; $\mathrm{kVp}=$ kilovoltage peak; $\mathrm{VMI}=$ virtual monochromatic image

$\mathbf{T}$ here are emerging applications of dual-energy CT $(\text { DECT })^{1}$ in all the major subspecialties of radiology. ${ }^{2-8}$ Studies have increasingly been demonstrating potential advantages of DECT for the evaluation of head and neck pathologies. ${ }^{8-18}$ Extrapolating from abdominal imaging, 70-keV virtual monochromatic image

Received December 18, 2014; accepted after revision January 31, 2015.

From the Department of Radiology (S.L., M.L., R.F.), Jewish General Hospital, McGill University, Montreal, Quebec, Canada; Department of Radiology (R.G.), Massachusetts General Hospital, Harvard Medical School, Boston, Massachusetts; Joint Department of Medical Imaging (E.Y.), Princess Margaret Hospital, University Health Network, University of Toronto, Toronto, Ontario, Canada; and Department of Radiology (H.D.C.), Massachusetts Eye and Ear Infirmary, Harvard Medical School, Boston, Massachusetts.

Abstract previously presented at: Annual Meeting of the American Society of Head and Neck Radiology, September 10-14, 2014; Seattle, Washington. S.L. received the American Society of Head and Neck Radiology's Radiologist-in-Training award.

Please address correspondence to Reza Forghani, MD, PhD, Department of Radiology, Jewish General Hospital, Room C-212.1, 3755 Cote Ste-Catherine Rd, Montreal, Quebec H3T 1E2, Canada; e-mail: rforghani@jgh.mcgill.ca

-- Indicates open access to non-subscribers at www.ajnr.org

http://dx.doi.org/10.3174/ajnr.A4314
(VMI) reconstructions are believed to be those that most closely resemble a standard single-energy CT acquisition ${ }^{19}$ and are usually the default setting for CT of the neck. On the other hand, enhancing tumors have increased attenuation on lower-kiloelectron volt (keV) VMIs, closer to the $k$ edge of iodine, ${ }^{20}$ albeit at the expense of other factors such as increased image noise. A recent study using a dual-source system (Somatom Definition Flash; Siemens, Erlangen, Germany) evaluated extrapolated monoenergetic datasets at 40,60, 80, and $100 \mathrm{keV}$, and the authors concluded that image reconstructions at $60 \mathrm{keV}$ improved lesion enhancement and the contrast-to-noise ratio (CNR), subjective overall image quality, and tumor delineation in head and neck squamous cell carcinoma (HNSCC). ${ }^{10}$

Most studies that evaluated HNSCC, other than a study that evaluated DECT for the differentiation of benign and malignant tumors, ${ }^{14}$ were performed by using dual-source CT. The other major system currently in clinical use is a single-source singledetector DECT system. This system is based on rapid kilovoltage peak $(\mathrm{kVp})$ switching that enables near-simultaneous acquisition of high- and low-energy projection data (GE Discovery 


\begin{tabular}{lc}
\multicolumn{1}{c}{ Tumor Type } & $\begin{array}{c}\text { No. of } \\
\text { Patients }\end{array}$ \\
\hline Untreated $(n=$ 22), primary site & 7 \\
Larynx & 1 \\
Hypopharynx & 3 \\
Retromolar trigone, anterior tonsillar pillar & 5 \\
Oral cavity-other & 3 \\
Oropharynx-other & 3 \\
Sinuses, nose & \\
Recurrent or metastatic $(n=8)$ & 2 \\
Oral cavity & 1 \\
Oropharynx & $5^{\mathrm{a}}$ \\
Other & \\
\hline
\end{tabular}

${ }^{a}$ Head and neck squamous cell carcinoma invading the parotid gland, $n=2$; parapharyngeal space metastasis, $n=1$; cheek, $n=1$; and neopharynx, $n=1$.

CT750HD; GE Healthcare, Milwaukee, Wisconsin). In this system, spectral separation is achieved on the basis of projectionbased material decomposition by using the fast sampling capabilities of a proprietary scintillator detector with low afterglow. ${ }^{5,21}$ Although the broad principles behind both DECT acquisition systems are similar, there are significant differences in hardware, acquisition, and postprocessing. As a result, any cross-platform application of observations made by using either system requires validation. Furthermore, there is currently no consensus on the optimal VMIs for the evaluation of HNSCC or the approach to incorporate DECT into routine clinical use.

The hypothesis behind our investigation was that VMIs acquired at energies other than $70 \mathrm{keV}$, either alone or in combination, can enhance the conspicuity of HNSCC. The objective of this investigation, therefore, was to determine the optimal VMI that provides the highest image quality and the VMI that enables optimal tumor visualization by using a single-source DECT scan with rapid $\mathrm{kVp}$ switching. This determination was made by objectively and quantitatively analyzing normal structures at different levels in the neck and tumors at different primary sites. Spectral evaluation was performed by using different VMI energy levels ranging from 40 to $140 \mathrm{keV}$ in 5-keV increments, and mean attenuation, SNR, and CNR were used as end points for evaluation.

\section{MATERIALS AND METHODS \\ Patients}

This study was approved by the institutional review board at the Jewish General Hospital (Montreal, Quebec, Canada). A total of 40 patients who had undergone DECT between June 2013 and July 2014 were evaluated retrospectively. There were 10 consecutive healthy patients and 30 consecutive patients with histopathologically proven (by biopsy and/or surgery) HNSCC who met the selection criteria discussed below. ${ }^{18}$ Normal cases consisted of normal or near-normal scan results with minor incidental findings (dental periapical lucencies, benign reactive lymph nodes or tonsillar enlargement, and incidental cutaneous lesions such as sebaceous cysts) in patients without known malignancy or major systemic disease. To have a broad and representative sample of HNSCC, patients with primary untreated or recurrent/metastatic tumors from different sites were included (Table). Exclusion criteria included suspected HNSCC not confirmed by biopsy or sur- gery and any tumor that was too small for sampling by the minimum preset ROI size and numbers (see below).

\section{CT Technique}

Each patient was scanned with the same 64-section dual-energy scanner (Discovery CT750HD; GE Healthcare). Examinations were performed after the administration of $80 \mathrm{~mL}$ of iopamidol (Isovue 300; Bracco Diagnostics, Princeton, New Jersey) injected at a rate of $2 \mathrm{~mL} / \mathrm{s}$ and the patients were scanned after a delay of 65 sec. All scans were acquired in dual-energy rapid 80 - to $140-\mathrm{kVp}$ switching mode by using the following gemstone spectral imaging protocol: gemstone spectral imaging preset at 15, a large-scan field of view (up to $50 \mathrm{~cm}$ ), a $40-\mathrm{mm}$ beam collimation, a 0.6 second rotation time, and a 0.984:1 helical pitch. Images were reconstructed into $1.25-\mathrm{mm}$ sections in a $25-\mathrm{cm}$ display field of view and a $512 \times 512$ matrix. The average CT dose index volume for the main acquisition (entire neck to carina) was $17.3 \mathrm{mGy}$.

\section{Postprocessing and Image Analysis}

Postprocessing and General Analysis. A 70-keV reconstruction is generated automatically by the scanner for standard clinical use. Quantitative image analysis was performed at an Advantage 4.6 workstation (GE Healthcare). Normal structures or lesions were evaluated with circular ROIs, and CT attenuation (in Hounsfield units) and standard deviation were measured within the ROIs. In each case, quantitative spectral analysis was performed in identical ROIs at different VMI energy levels ranging from 40 to 140 $\mathrm{keV}$, in $5-\mathrm{keV}$ increments, for a total of 21 energy levels per ROI. Each normal structure or lesion was evaluated with multiple ROIs (described in greater detail below). For each patient, the mean attenuation of a given structure or lesion was determined on the basis of the average Hounsfield units of the respective ROIs in that structure or lesion. Image noise was based on the SD in an ROI, and the average noise for each structure or lesion was calculated by obtaining the average SD in their respective ROIs. ${ }^{5}$ As described in greater detail below, because of different sizes of normal structures and lesions, different-sized ROIs had to be used. So that the results would not be biased toward larger structures (eg, larger tumors), the average ROI for a given normal structure or lesion for each patient was given equal weight when pooling data from multiple patients.

Spectral Evaluation of SNR in Normal Tissues. In the first part of the study, the SNR of muscles and major glands was evaluated. Because of changes in tissue composition and the shape of the neck in the craniocaudal plane, muscles were evaluated at 6 different levels in the neck. From cranial to caudal, the lateral pterygoid (level of fossa of Rosenmüller), masseter (level of parotid), genioglossus (oral cavity), sternocleidomastoid muscle at the level of the submandibular glands, sternocleidomastoid muscle at the level of true vocal cords, and sternocleidomastoid muscle at the level of the thyroid gland were evaluated. In addition, the parotid, submandibular, sublingual, and thyroid glands were evaluated. For consistency, the right side of the neck was evaluated, except for the sublingual gland and genioglossus muscle. In these cases, because of their relatively small sizes, the side with the larger gland or both sides were evaluated. Normal-structure ROIs were iden- 


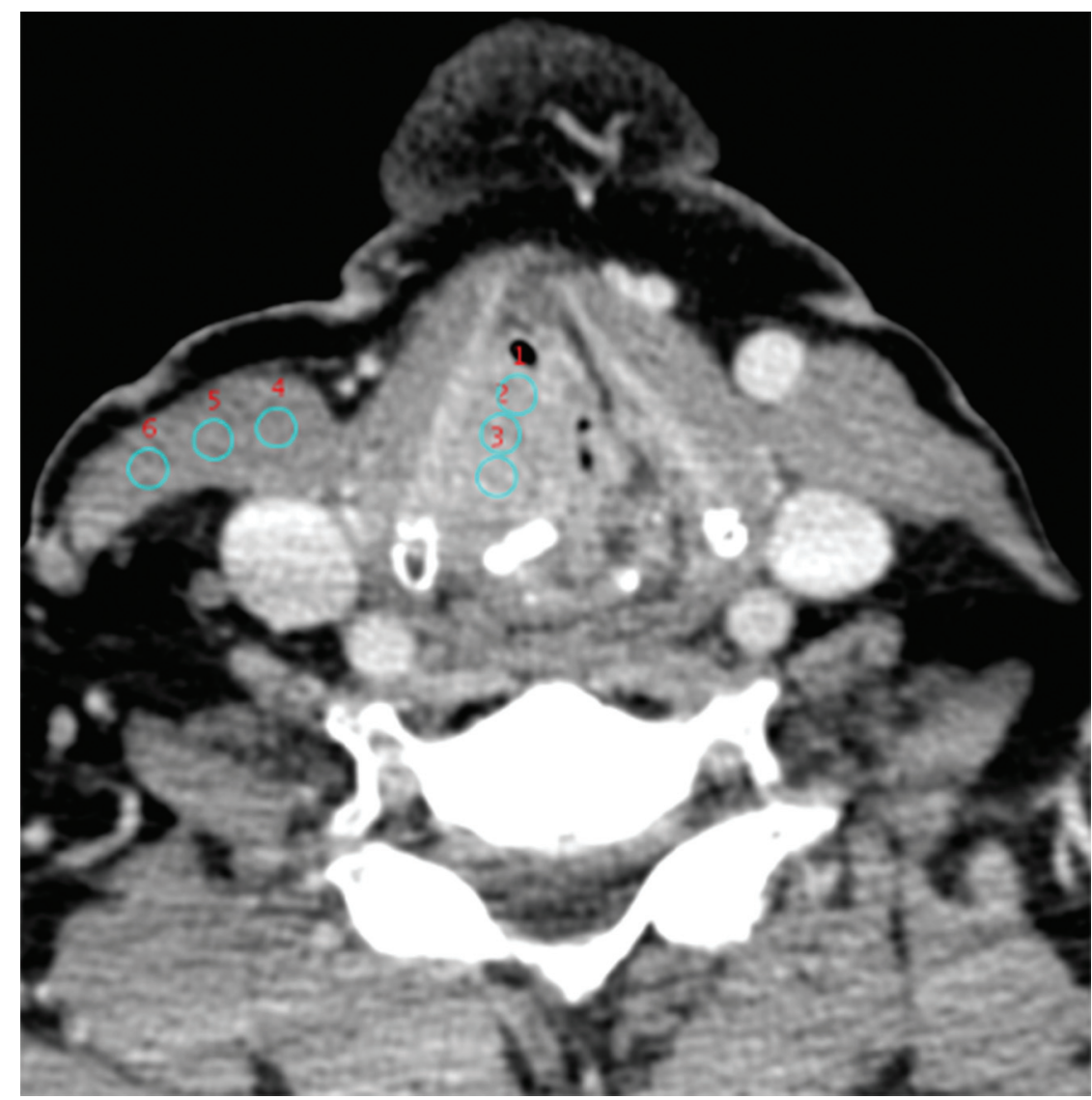

FIG 1. Example of a 5-mm ROI used for the assessment of laryngeal cancer and nearby sternocleidomastoid muscle. As described in detail in the text, in patients with head and neck squamous cell carcinoma, each lesion and a nearby structure were evaluated with 9 nonoverlapping ROIs on multiple sections.

tified by S.L. (diagnostic radiology resident with 3 years of training) and reviewed by R.F (attending physician with fellowship training and 4 years postfellowship experience in neuroradiology and head and neck radiology) before recording the data for further analysis. For each structure, 3 nonoverlapping ROIs were placed. When possible, all 3 ROIs were placed on the same section, except those for small structures (sublingual and genioglossus), for which the ROIs had to be placed on more than 1 section to obtain good coverage and avoid overlaps or artifacts. ROI sizes that enabled good sampling without overlapping or volume averaging with adjacent structures were used. Areas of visible artifacts, such as from dental amalgam, were avoided. For this part of the study, 30 ROIs per patient were evaluated. Depending on the size of the structure being evaluated, the minimum individual ROI diameter used was $3.3 \mathrm{~mm}$ (corresponding to a sampled area per ROI of $8.6 \mathrm{~mm}^{2}$ ), and the maximum diameter used was $5.8 \mathrm{~mm}$ (corresponding to a sampled area per ROI of $26.4 \mathrm{~mm}^{2}$ ). The SNR was calculated for each structure, in each patient, by dividing the mean CT attenuation for the 3 ROIs by the mean noise (SD) for the ROIs.

Spectral Evaluation of Tumors and Pathologic Lymph Nodes. For tumors, the mean attenuation and tumor-muscle CNR were calculated. In each patient, the tumor and a nearby normal muscle (completely separate from tumor without contact or invasion of any part of that muscle) were evaluated. Each tumor or normal muscle was evaluated with a total of 9 ROIs, placed on at least 3 separate sections (Fig 1). In addition, 17 pathologic lymph nodes in 9 of the patients were evaluated, and each node was evaluated with 9 ROIs. Only grossly pathologic nodes were evaluated on the basis of either 1) biopsy or neck dissection, when such results were available, or 2) an abnormal-appearing node in the primary or secondary drainage area of the primary tumor by at least 2 criteria among the following: abnormal short-axis diameter, internal necrosis/heterogeneity, rounded contour, or irregular contour. ${ }^{22}$ For tumors and lymph nodes, the ROIs were placed in the homogeneous-appearing enhancing part of the lesion, avoiding areas of cystic change/ necrosis or visible artifacts. For muscles, the ROIs were placed in areas without visible artifacts. For each normal-appearing structure or lesion, the mean attenuation was determined on the basis of the average Hounsfield units of the respective ROIs in that structure or lesion. Image noise was calculated as the $\mathrm{SD}$ in the ROI, and the average noise for each structure or lesion was calculated by obtaining the average SD in their respective ROIs. ${ }^{5}$ For tumors, the minimum individual ROI diameter used was $1.7 \mathrm{~mm}$ (sampled area per ROI, $2.3 \mathrm{~mm}^{2}$ ), and the maximum diameter used was $5.5 \mathrm{~mm}$ (sampled area per ROI, $23.8 \mathrm{~mm}^{2}$ ). For lymph nodes, the ROI size range was $2.3-4.7 \mathrm{~mm}$ (area, $4.2-17.3 \mathrm{~mm}^{2}$ ), and for muscles it was $2.3-4.8 \mathrm{~mm}$ (area, $4.2-18.1 \mathrm{~mm}^{2}$ ). To select an ROI size that provided good sampling and could be applied to different structures in each patient, the size of the ROIs varied because of differences in lesion or muscle size, tumor or lymph node homogeneity, and presence of artifacts. All ROIs in this section were placed by the attending head and neck radiologist (R.F.). The CNR was calculated individually for tumor or lymph node in each patient by using the formula CNR $=$ (average lesion attenuation average muscle attenuation $) / \mathrm{V}$ (variance [lesion] + variance [muscle]). ${ }^{5}$

\section{Statistical Analysis}

The results are reported as mean \pm SD. All comparisons between patients were performed by using the mean attenuation and/or noise from the structure or lesion of interest from each patient (and not by simply pooling all individual ROIs among all patients, which would have artificially inflated the statistical power of the study). Means from 2 different groups were compared by using a Student $t$ test. For comparisons of multiple ( $>2$ ) groups, 1-way ANOVA with the Dunnett multiple-comparisons test was used. Data from different ROI samples were compared by using an un- 

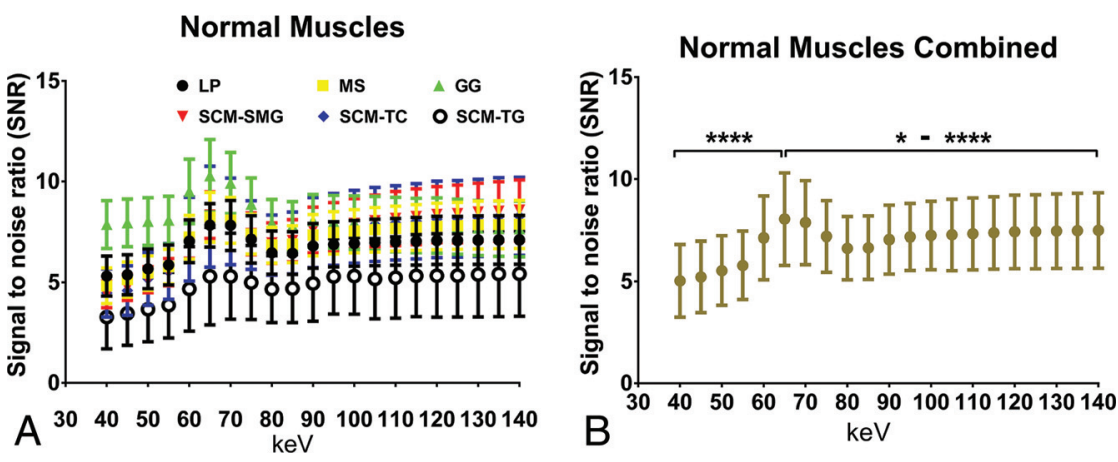

FIG 2. Quality index across the range of VMIs in muscles at different levels in the neck. Signalto-noise ratios are shown for the lateral pterygoid (LP, level of fossa of Rosenmuller), masseter (MS, level of parotid and masticator space), genioglossus (GG, oral cavity), or sternocleidomastoid (SCM-SMG, level of submandibular glands; SCM-TC, level of true vocal cords; SCM-TG, level of thyroid gland) $(A)$ and for all muscles combined $(B)$. The highest SNR for the individual muscle curves $(A)$ and data from all the muscles combined $(B)$ was on VMls reconstructed at $65 \mathrm{keV}(n=$ 10; total ROIs evaluated, 180). ${ }^{*} P<.05 ;{ }^{\star \star \star *} P<.0001$ (1-way ANOVA).
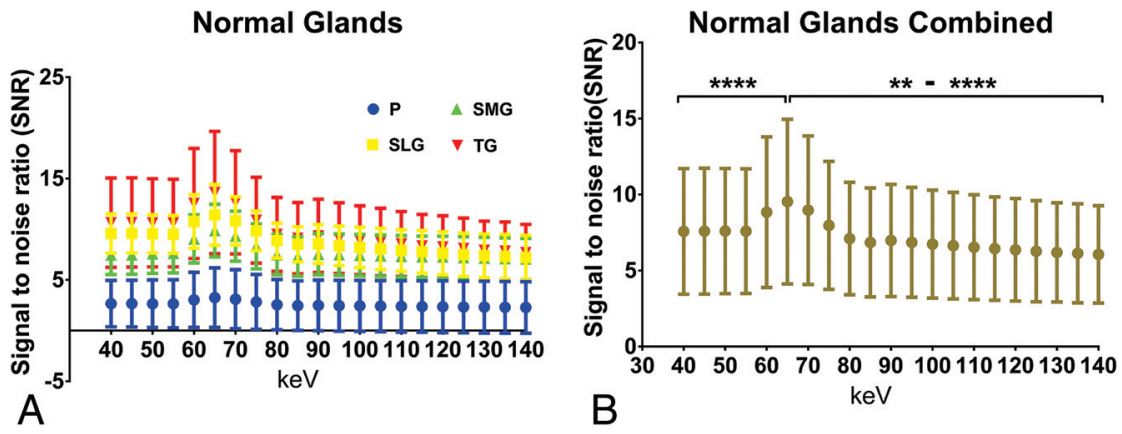

FIG 3. Quality index across the range of VMIs in glands at different levels in the neck. Signal-tonoise ratios are shown for the parotid gland (P), sublingual gland (SLG), submandibular gland (SMG), and thyroid gland (TG) $(A)$ and all glands combined $(B)$. The highest SNR for the individual muscle curves $(A)$ and data from all the glands combined $(B)$ was on VMls reconstructed at $65 \mathrm{keV}$ ( $n=10$; total ROls evaluated, 120). ** $P<.01 ;{ }^{* * *} P<.0001$ (1-way ANOVA).

SNR - Tumor and Pathologic Lymph Nodes

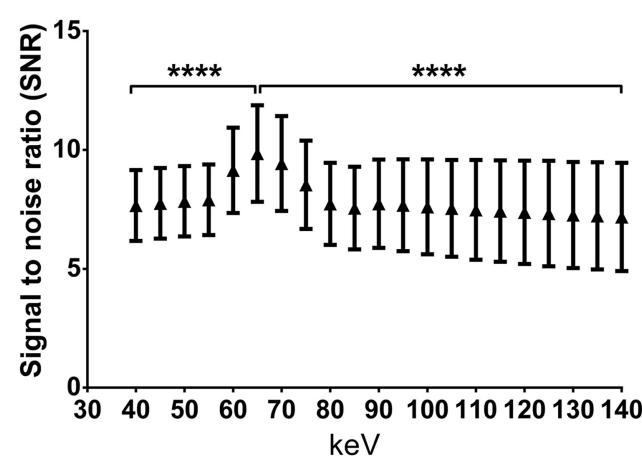

FIG 4. Quality index across the range of VMIs in HNSCC and metastatic lymph nodes. Combined SNRs from 30 tumors and 17 pathologic lymph nodes show that the highest SNR is at $65 \mathrm{keV}$. ${ }^{* \star \star *} P<$ .0001 (1-way ANOVA).

paired test. Spectral data at different keVs derived from the same ROIs were analyzed by using paired analysis. Variance was calculated by using the standard formula variance $=\mathrm{SD}^{2}$. A $P$ value of $<.05$ was considered statistically significant. We used GraphPad Prism software for statistical analysis (version 6.005; GraphPad Software, San Diego, California).

\section{RESULTS}

DECT scans from 10 healthy subjects and 30 subjects with HNSCC with a total of 993 ROIs were evaluated. The mean patient age was 66 years (range, 37-97 years; 20 women, 20 men). In healthy subjects, a total of 6 muscle levels and 4 glands per patient were evaluated, each with 3 ROIs, corresponding to a total of 300 ROIs. The average ROI area evaluated per structure was $62.7 \mathrm{~mm}^{2}$ (range, $27.2-75.6 \mathrm{~mm}^{2}$ ). In the HNSCC group, tumors, pathologic lymph nodes, and reference muscles were evaluated with a total of 270,153 , and 270 ROIs, respectively. The average ROI area evaluated was $105.7 \mathrm{~mm}^{2}$ (range, 21.2$212.1 \mathrm{~mm}^{2}$ ) for tumors, $71.0 \mathrm{~mm}^{2}$ (range, $37.7-151.8 \mathrm{~mm}^{2}$ ) for lymph nodes, and $126.3 \mathrm{~mm}^{2}$ (range, 37.7-212.1 $\mathrm{mm}^{2}$ ) for muscles.

\section{Spectral Attenuation Characteristics and Optimal SNR of Muscles and Normal Glands}

Muscle and gland attenuation progressively increased on lower-keV VMIs. However, the highest SNR for all the muscle groups and glands was at $65 \mathrm{keV}$. For all muscles combined, the mean SNR was 8.0, and for all glands combined the mean SNR was 9.5 at $65 \mathrm{keV}$. At this energy, the SNR was significantly greater than those at all other energies (1-way ANOVA with Dunnett multiplecomparisons test; Figs 2 and 3). This SNR was closely matched by the SNR at $70 \mathrm{keV}$ (muscles, 7.9; glands, 9.0), followed by that at $60 \mathrm{keV}$ (muscles, 7.1; glands, 8.8; Figs $2 B$ and $3 B$ ). In addition to normal tissues, we also evaluated the SNRs of 30 tumors and 17 pathologic lymph nodes, and the optimal SNR was also at $65 \mathrm{keV}$ (Fig 4).

\section{Optimal VMIs and CNR for Evaluation of HNSCC}

Similar to muscle, tumor attenuation increased at lower keVs. Tumor attenuation was highest on 40-keV VMIs, significantly different from those at all other energy levels (1-way ANOVA with Dunnett multiple-comparisons test; Fig 5). However, the slope of the increase in tumor attenuation was greater than that in muscles, resulting in greater attenuation separation in the low-keV range (Fig 5A). The greatest difference between tumor and muscle attenuation was at $40 \mathrm{keV}$. On VMIs reconstructed at this energy level, the mean tumor attenuation was $207.9 \pm 46.8$ and the mean muscle attenuation was $115.2 \pm 31.3(P=.03$, unpaired 2 -tailed $t$ test; Fig 5$)$. Because there is a progressive increase in image noise on lower-keV images, we also calculated tumormuscle CNR as a quantitative index for lesion conspicuity. The tumor CNR was likewise highest at $40 \mathrm{keV}$, despite the increase in image noise, and significantly higher than those at other 

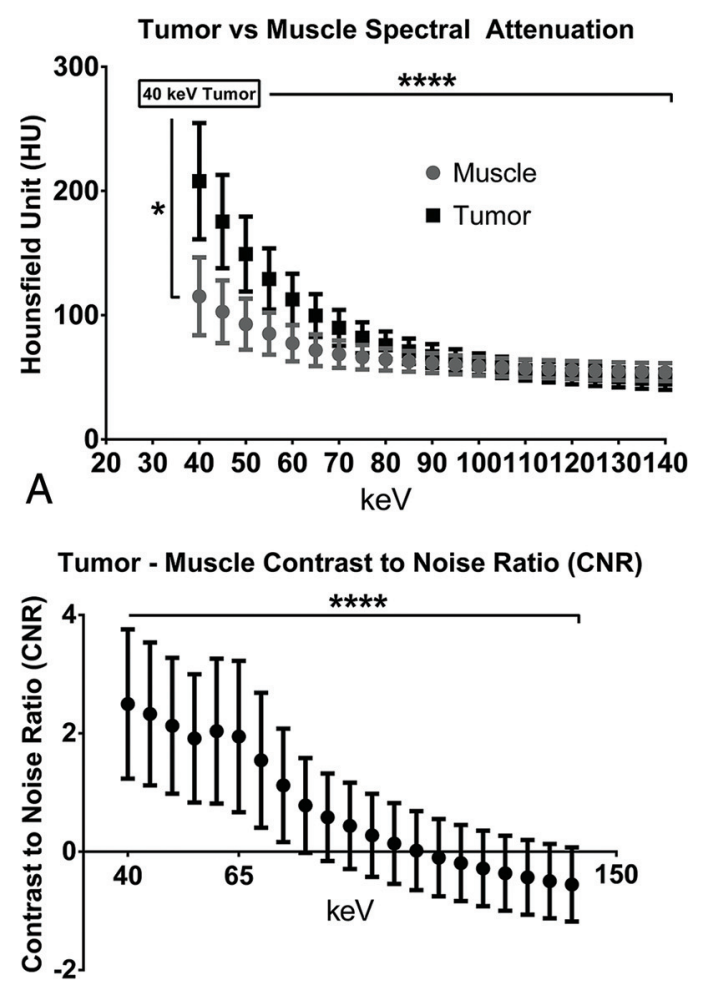

$\mathrm{B}$

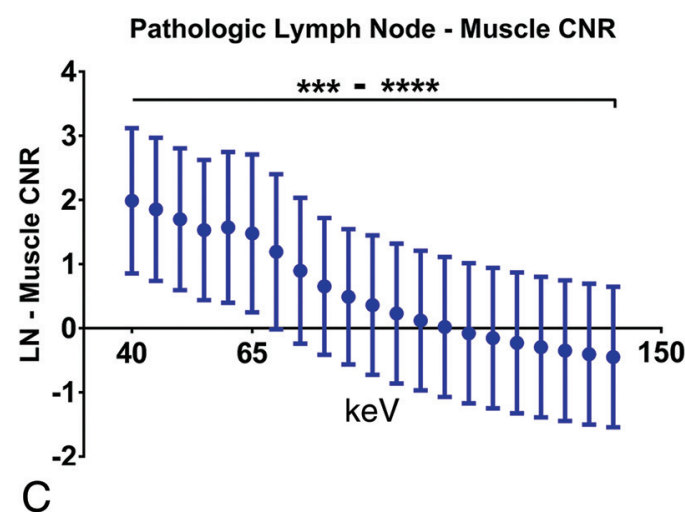

FIG 5. Optimal virtual monochromatic energy level for evaluation of HNSCC and pathologic lymph nodes. A, Spectral Hounsfield unit curves of HNSCC compared with those of muscle $(n=30)$. The highest tumor attenuation was at $40 \mathrm{keV}$, with a statistically significant difference compared with all other energy levels and with mean muscle attenuation at $40 \mathrm{keV}$. B, Tumor-muscle CNR $(n=30)$. The CNR was highest at $40 \mathrm{keV}$, significantly different from those at all other energy levels. C, Pathologic lymph node-muscle CNR $(n=17)$. The CNR was highest at $40 \mathrm{keV}$, significantly different from those of all other energy levels. ${ }^{\star \star} P<.01 ;{ }^{* \star \star} P<.0001$ (1-way ANOVA).

energy levels (1-way ANOVA with Dunnett multiple-comparisons test; Fig 5).

\section{Optimal VMI for Evaluation of Metastatic Lymphadenopathy}

A total of 17 metastatic lymph nodes were evaluated, and lymph node-muscle CNRs were calculated as measures of conspicuity. Similar to those of tumors, the lymph node-muscle CNR was highest at $40 \mathrm{keV}$ and significantly different from those at other energy levels (1-way ANOVA with Dunnett multiple-comparisons test; Fig 5). Although tumor-muscle and lymph node-mus- cle CNRs were highest at $40 \mathrm{keV}$, the optimal SNR was at $65 \mathrm{keV}$, similar to that of normal tissues (Fig 4).

\section{Comparison of 40-keV VMIs with Other Key DECT VMIs}

Mean tumor attenuation on $40-\mathrm{keV}$ VMIs was significantly higher than that on 60-keV VMIs, 65-keV VMIs (optimal tissue SNR), and 70-keV VMIs (current standard reconstruction) $(P<$ .0001; Fig 6A). Tumor-muscle CNRs were likewise significantly higher on 40-keV VMIs than on 60-, 65-, and 70-keV VMIs $(P<$ .0001; Fig 6B). Qualitatively, the higher attenuation and CNR are result in increased tumor conspicuity at $40 \mathrm{keV}$ (Fig 7).

\section{DISCUSSION}

In this investigation, we evaluated the optimal VMI energy levels for evaluation of the neck. Normal structures and lesions were evaluated by a general quality index, the SNR. In addition, the VMI energy level that provided optimal HNSCC and pathologic lymph node conspicuity was quantitatively evaluated by measuring lesion attenuation and calculating the CNR. Currently, the default reconstruction on the single-source dual-energy scanner with rapid $\mathrm{kVp}$ switching used in this study for neck CTs is $70 \mathrm{keV}$, the VMI believed to simulate the standard $120-\mathrm{kVp}$ single-energy acquisition by extrapolation from abdominal CT studies. On the basis of our results, the $65-\mathrm{keV}$ VMI has the optimal SNR and can be used as the default reconstruction for assessment of the neck. Similar observations have been made for head DECT scans by using this type of scanner. ${ }^{5}$ By extrapolation, one would expect that the $65 \mathrm{keV}$ VMI also provides the optimal SNR for evaluation of other normal soft-tissue structures in the neck, such as small normal-appearing lymph nodes, but this could be validated in future studies targeted at the evaluation of those structures.

Although the 65-keV VMIs yielded the best SNR, both absolute tumor attenuation and contrast (by using normal muscles as reference) were highest on 40-keV VMIs. The increase in attenuation on lower-keV VMIs is expected, because these energies approach the $k$ edge of iodine. Although image noise increases with decreasing VMI energy levels, the tumor-muscle CNR was still highest on the $40-\mathrm{keV}$ reconstructions. This observation is different from that in a recently published study in which 40-, 60-, 80-, and 100-keV VMIs were evaluated, and it was reported that the highest tumor-muscle CNR was achieved at $60 \mathrm{keV} .{ }^{10}$ The reason for the difference is not entirely clear, but a number of explanations need to be considered. In the aforementioned study, a dualsource scanner was used. Apart from differences in acquisition, the methods of postprocessing for that scanner are different. Therefore, one possibility is that the differences are technical, related either to the different modes of acquisition and/or postprocessing algorithms. It is also noteworthy that noise was measured differently by using an ROI outside the patient, placed in air. We prefer using the SD within the tissues of interest as an indicator of noise and believe that it is more pertinent to clinical evaluation, similar to the method used by Pomerantz et al. ${ }^{5}$ This represents another potential source of variation, though we believe that it is less likely to account for the differences between the 2 studies. Future studies using larger sample sizes and comparing both systems would be of interest.

In our study, we evaluated normal structures at multiple levels 

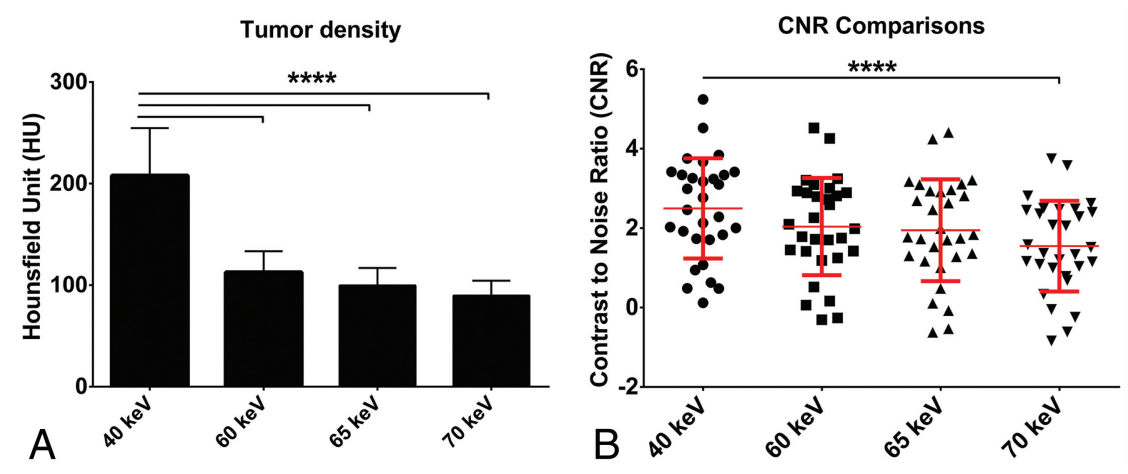

FIG 6. Optimal tumor attenuation and CNR comparisons. $A$, Tumor attenuation comparison of 40-keV VMls with those on 60-, 65-, and 70-keV VMIs $(n=30)$. B, Scatterplot of CNRs comparing 40-keV VMIs with 60-, 65-, and 70-keV VMl reconstructions. ${ }^{\star * * *} P<.0001$.
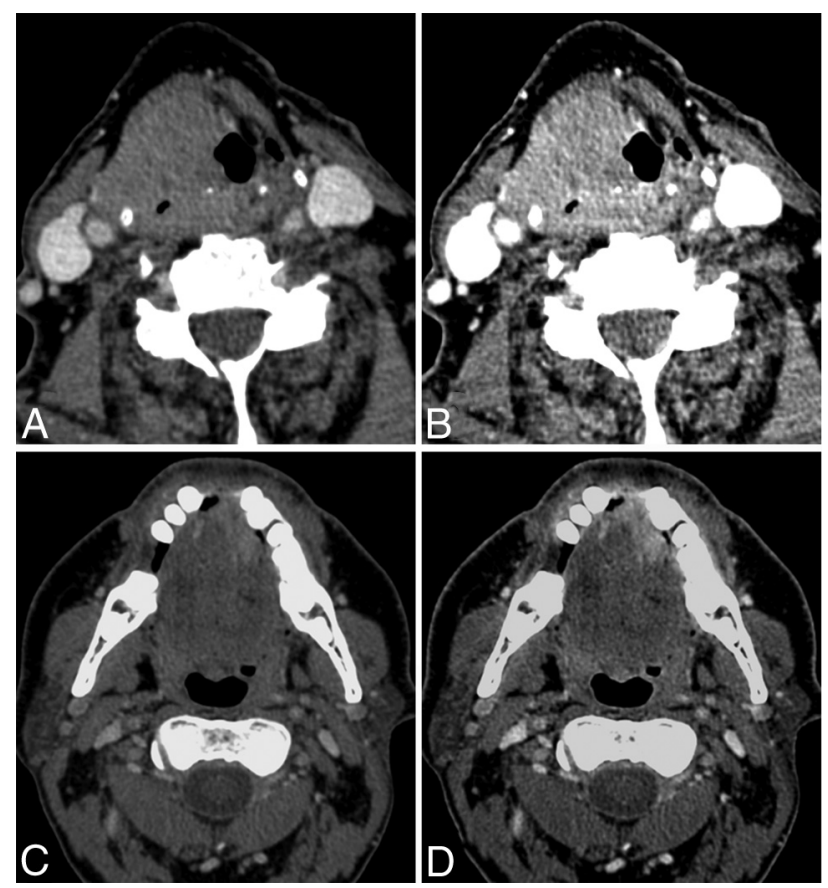

FIG 7. Case examples comparing the standard 70-keV VMI reconstructions with $40 \mathrm{keV}$ VMls. $A$ and $B$, A 92-year-old man with supraglottic squamous cell carcinoma. The same VMls reconstructed at 70 $\mathrm{keV}(A)$ and $40 \mathrm{keV}(B)$ and with similar windowing (compare subcutaneous fat) are shown. There is increased tumor conspicuity and better visualization of the tumor interface with adjacent prelaryngeal strap muscle. $C$ and $D, A$ 60-year-old man with floor-of-mouth squamous cell carcinoma. VMls reconstructed at $70 \mathrm{keV}(C)$ and $40 \mathrm{keV}(D)$ are shown. Note the increased tumor conspicuity on the 40-keV VMI reconstruction.

in the neck and tumors at different subsites to make sure that our conclusions can be applied generally to the evaluation of the neck. However, one limitation is that the number of HNSCC tumors for each specific subsite was small, and our study did not evaluate lesions centered at the skull base. Therefore, it is possible that additional adjustments may further improve evaluation at a given cancer subsite. Furthermore, we focused on the enhancing part of the tumor rather than the hypoenhancing core, which is pertinent for distinguishing the tumor-normal tissue interface. However, there are additional parameters of clinical interest that were not evaluated in this study, such as distinguishing enhancing and hypoenhancing parts of a lesion, which can potentially be relevant

\section{CONCLUSIONS}

for the evaluation of lesions such as small pathologic lymph nodes. These topics are of interest for future research.

We also focused on quantitative objective evaluation, which we believe is more robust and reliable than subjective evaluation. It has been our experience that similarly windowed low-keV VMIs are clearly distinguishable from the standard-energy VMIs at $70 \mathrm{keV}$, which makes a blinded subjective comparison nearly impossible. Furthermore, we have found that user acceptance of noise levels changes with exposure and experience. Therefore, although subjective evaluation is an interesting topic of future investigation, any such study needs to be designed carefully with well-defined end points and by taking into account the above-mentioned considerations and pitfalls. Last, our study did not address the optimal assessment of areas obscured by artifacts. Artifact reduction is a complex topic that merits a separate dedicated investigation.

The optimal image SNR is at $65 \mathrm{keV}$, but tumor conspicuity, compared with that of other soft tissues, is greatest at $40 \mathrm{keV}$. Therefore, on the basis of our observations, we recommend that standard neck reconstructions using this type of scanner be made with a $65-\mathrm{keV}$ VMI and in addition, a $40-\mathrm{keV}$ VMI reconstruction generated for the evaluation of patients with HNSCC. We do not advocate replacing the $65-\mathrm{keV}$ reconstruction with the $40-\mathrm{keV}$ VMI. Instead, we recommend that both the standard 65-keV VMI reconstructions and the $40-\mathrm{keV}$ VMIs be automatically generated and sent to the PACS for evaluation of patients with cancer. Optimal evaluation of the neck may then be performed by a multiparametric approach. Using the proposed approach, the $65-\mathrm{keV}$ VMIs providing the best overall image quality are used for general evaluation of the neck, supplemented with the targeted use of 40-keV VMIs for tumor detection and optimal HNSCC-soft tissue boundary visualization.

\section{ACKNOWLEDGMENTS}

We thank Ms. Veronika Glyudza for her assistance in data preparation and processing.

Disclosures: Rajiv Gupta_UNRELATED: Board Membership: Actelion Corp, Comments: advising the company on imaging in their drug trials; Grants/Grants Pending: Siemens Medical Solutions, ${ }^{*}$ Comments: research grant to Massachusetts General Hospital; the principal investigator on the grant (Udo Hoffman, MD) is not an author on this paper; Travel/Accommodations/Meeting Expenses Unrelated to Activities Listed: Siemens Medical Solutions, Comments: travel support for an educational symposium on photon-counting CT organized by Siemens Medical Solutions. Mark Levental-UNRELATED: Consultancy: Biogen Idec, Comments: honorarium for attending a training seminar; Travel/Accommodations/Meeting Expenses Unrelated to Activities Listed: Royal College Examiner, Comments: reimbursement of expenses. Hugh Curtin-UNRELATED: Royalties: Elsevier, Comments: royalties for textbook. Reza Forghani-UNRELATED: Consultancy: Biogen Idec, Collège des médecins du Québec, Comments: one-time honorarium for attending a training seminar; expert consultant for Collège des médecins du Québec (telemedicine committee, quality assurance); Expert Testimony: Collège des médecins du Québec, Comments: quality assurance and reviews; Payment for Lectures (including service on Speakers Bu- 
reaus): Peer Review for Departmental QA and Audits: Essentials of a Successful Program, Benchmarks for Evaluation, and Challenges (Ontario Association of Radiologists CME course, 2014); Sinus Anatomy: Normal and Postoperative (Ontario Association of Radiologists CME course, 2014); Visiting Professor, McMaster University Health Sciences Center; Radiology Grand Rounds, St. Joseph's Healthcare Hamilton, 2013; Stock/Stock Options: Real Time Medical, Comments: member, medical and quality assurance committee. *Money paid to the institution.

\section{REFERENCES}

1. Johnson TRC, Kalender WA. Physical background. In: Johnson T, Fink C, Schönberg SO, et al, eds. Dual Energy CT in Clinical Practice. Berlin-Heidelberg: Springer-Verlag; 2011:3-9

2. De Cecco CN, Darnell A, Rengo M, et al. Dual-energy CT: oncologic applications. AJR Am J Roentgenol 2012;199:S98-105

3. Heye T, Nelson RC, Ho LM, et al. Dual-energy CT applications in the abdomen. AJR Am J Roentgenol 2012;199:S64-70

4. Lu GM, Zhao Y, Zhang LJ, et al. Dual-energy CT of the lung. AJR Am J Roentgenol 2012;199:S40-53

5. Pomerantz SR, Kamalian S, Zhang D, et al. Virtual monochromatic reconstruction of dual-energy unenhanced head CT at $65-75 \mathrm{keV}$ maximizes image quality compared with conventional polychromatic CT. Radiology 2013;266:318-25

6. Postma AA, Hofman PA, Stadler AA, et al. Dual-energy CT of the brain and intracranial vessels. AJR Am J Roentgenol 2012;199: S26-33

7. Vliegenthart R, Pelgrim GJ, Ebersberger U, et al. Dual-energy CT of the heart. AJR Am J Roentgenol 2012;199:S54-63

8. Vogl TJ, Schulz B, Bauer RW, et al. Dual-energy CT applications in head and neck imaging. AJR Am J Roentgenol 2012;199:S34-39

9. Tawfik AM, Kerl JM, Bauer RW, et al. Dual-energy CT of head and neck cancer: average weighting of low- and high-voltage acquisitions to improve lesion delineation and image quality-initial clinical experience. Invest Radiol 2012;47:306-11

10. Wichmann JL, Nöske EM, Kraft J, et al. Virtual monoenergetic dualenergy computed tomography: optimization of kiloelectron volt settings in head and neck cancer. Invest Radiol 2014;49:735-41

11. Toepker M, Czerny C, Ringl H, et al. Can dual-energy CT improve the assessment of tumor margins in oral cancer? Oral Oncol 2014;50:221-27

12. Kuno H, Onaya H, Fujii S, et al. Primary staging of laryngeal and hypopharyngeal cancer: CT, MR imaging and dual-energy CT. Eur J Radiol 2014;83:e23-35

13. Kuno $\mathrm{H}$, Onaya $\mathrm{H}$, Iwata $\mathrm{R}$, et al. Evaluation of cartilage invasion by laryngeal and hypopharyngeal squamous cell carcinoma with dualenergy CT. Radiology 2012;265:488-96

14. Srinivasan A, Parker RA, Manjunathan A, et al. Differentiation of benign and malignant neck pathologies: preliminary experience using spectral computed tomography. J Comput Assist Tomogr 2013;37:666-72

15. Stolzmann P, Winklhofer S, Schwendener N, et al. Monoenergetic computed tomography reconstructions reduce beam hardening artifacts from dental restorations. Forensic Sci Med Pathol 2013; 9:327-32

16. Tawfik AM, Razek AA, Kerl JM, et al. Comparison of dual-energy CT-derived iodine content and iodine overlay of normal, inflammatory and metastatic squamous cell carcinoma cervical lymph nodes. Eur Radiol 2014;24:574-80

17. Tanaka R, Hayashi T, Ike M, et al. Reduction of dark-band-like metal artifacts caused by dental implant bodies using hypothetical monoenergetic imaging after dual-energy computed tomography. Oral Surg Oral Med Oral Pathol Oral Radiol 2013;115:833-38

18. Forghani R, Levental M, Gupta R, et al. Different spectral Hounsfield unit curve and high-energy virtual monochromatic image characteristics of squamous cell carcinoma compared with nonossified thyroid cartilage. AJNR Am J Neuroradiol 2015;36:1194-200

19. Patel BN, Thomas JV, Lockhart ME, et al. Single-source dual-energy spectral multidetector CT of pancreatic adenocarcinoma: optimization of energy level viewing significantly increases lesion contrast. Clin Radiol 2013;68:148-54

20. Krauss B, Schmidt B, Flohr TG. Dual source CT. In: Johnson T, Fink C, Schönberg SO, et al, eds. Dual Energy CT in Clinical Practice. Berlin-Heidelberg: Springer-Verlag; 2011:10-20

21. Chandra N, Langan DA. Gemstone detector: dual energy imaging via fast $\mathbf{k V p}$ switching. In: Johnson T, Fink C, Schönberg SO, et al, eds. Dual Energy CT in Clinical Practice. Berlin-Heidelberg: SpringerVerlag; 2011:35-41

22. Forghani R, Yu E, Levental M, et al. Imaging evaluation of lymphadenopathy and patterns of lymph node spread in head and neck cancer. Expert Rev Anticancer Ther 2015;15:207-24 